\title{
microRNA protects the heart
}

When the supply of oxygen to the heart is insufficient to meet metabolic demands, myocardial cells undergo apoptosis, which can cause myocardial infarction. Mitochondrial fission is known to play a part in this process, but the precise mechanisms involved have not been elucidated. Now, Wang et al. have delineated a key pathway mediating mitochondrion-dependent apoptosis in ischaemia that is regulated by the microRNA (miRNA) miR-499.

Having identified that miR-499 levels were reduced in the hearts of rats subjected to ischaemia, the authors showed that delivery of an miR-499 construct to mice decreased cardiomyocyte apoptosis in response to ischaemia. Furthermore, miR-499 knockdown in wild-type mice increased apoptosis and infarct size and decreased cardiac function. Thus, miR-499 seems to confer protection against cell death under low oxygen conditions.

Using a bio-informatics program, the authors identified both isoforms of the catalytic subunit of the phosphatase calcineurin - CNA $\alpha$ and $\mathrm{CNA} \beta$ - as potential targets of miR-499. In vivo, cardiac ischaemia increased the expression of CNA $\alpha$ and $\mathrm{CNA} \beta$, and this was attenuated by delivery of miR-499. Small interfering RNA constructs targeting both isoforms of calcineurin inhibited anoxia-induced apoptosis in vitro, highlighting a role for calcineurin in the cell death programme following oxygen deprivation.

It was previously known that dephosphorylation of the GTPase dynamin-related protein 1 (DRP1) by calcineurin promotes DRP1 translocation into mitochondria, where it causes scission of the outer membrane and mitochondrial fission. The authors hypothesized that this pathway might be involved in the apoptotic response to low oxygen. Indeed, exposure of cardiomyocytes to anoxia increased the abundance of DRP1 in mitochondria compared with the cytosol. Furthermore, knockdown of DRP1 reduced mitochondrial fragmentation and cell death in anoxic conditions in vitro, and reduced myocardial infarct size in vivo.

Importantly, the anoxiainduced accumulation of DRP1 in mitochondria and mitochondrial fragmentation were reduced by either knockdown of calcineurin or overexpression of miR-499. Furthermore, delivery of miR-499 attenuated cell death. Together, these results suggest a pathway in which miR-499 could reduce myocardial infarction by inhibiting calcineurin-dependent trafficking of DRP1 to mitochondria, preventing mitochondrial fission and cell death. The transcription factor $\mathrm{p} 53$, which is known to contribute to myocardial infarction, was also identified as a negative regulator of miR-499.

These findings identify key players in the anoxia-induced cell death pathway and highlight the therapeutic potential of upregulating miR-499 to reduce the damaging effects of myocardial infarction.

Katie Kingwell

ORIGINAL RESEARCH PAPER Wang, J-. X. et al. miR-499 regulates mitochondrial dynamics by targeting calcineurin and dynamin-related protein-1. Nature Med. 17, 71-78 (2011)

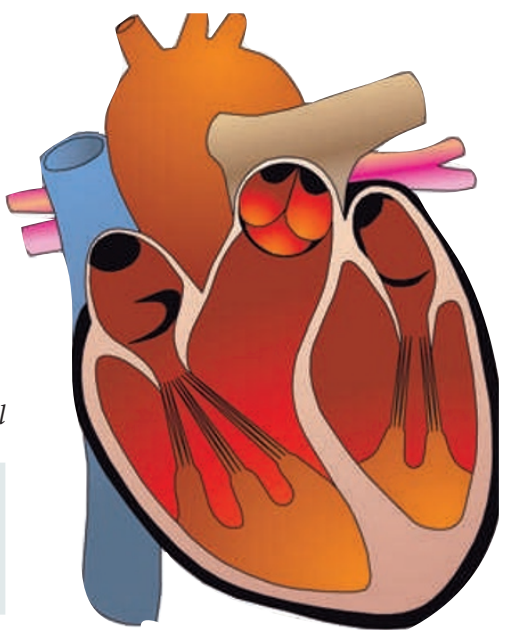

